\title{
Chitosan-based nanoparticles for improved anticancer efficacy and bioavailability of mifepristone
}

\author{
Huijuan Zhang ${ }^{1}$, Fuqiang $\mathrm{Wu}^{1}$, Yazhen $\mathrm{Li}^{1}$, Xiping Yang ${ }^{1}$, Jiamei Huang ${ }^{1}$, Tingting $\mathrm{Lv}^{1}$, \\ Yingying Zhang ${ }^{1}$, Jianzhong Chen ${ }^{*}$, Haijun Chen ${ }^{1}$, Yu Gao ${ }^{* 1}$, Guannan Liu ${ }^{3}$ \\ and Lee $\mathrm{Jia}^{1}$
}

\section{Full Research Paper}

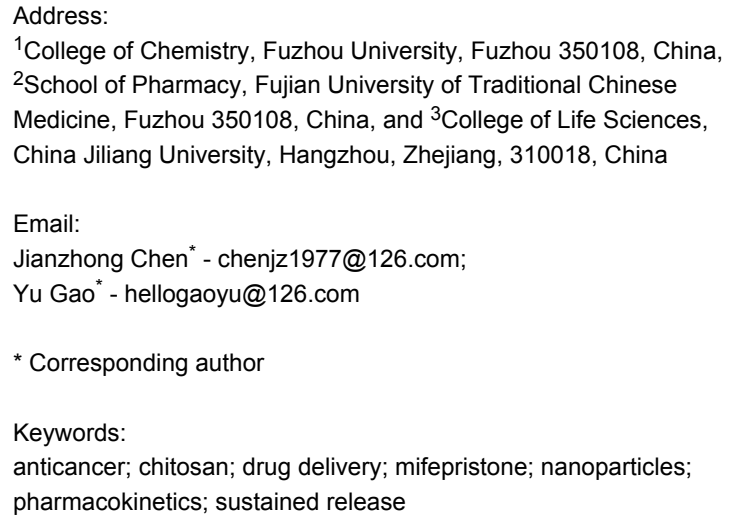

${ }^{1}$ College of Chemistry, Fuzhou University, Fuzhou 350108, China,

${ }^{2}$ School of Pharmacy, Fujian University of Traditional Chinese Medicine, Fuzhou 350108, China, and ${ }^{3}$ College of Life Sciences, China Jiliang University, Hangzhou, Zhejiang, 310018, China

\section{Email:}

Jianzhong Chen * - chenjz1977@126.com;

Yu Gao* - hellogaoyu@126.com

* Corresponding author

Keywords:

anticancer; chitosan; drug delivery; mifepristone; nanoparticles; pharmacokinetics; sustained release

\author{
Beilstein J. Nanotechnol. 2016, 7, 1861-1870. \\ doi:10.3762/bjnano.7.178 \\ Received: 28 July 2016 \\ Accepted: 06 November 2016 \\ Published: 28 November 2016 \\ Associate Editor: M. Stenzel \\ (C) 2016 Zhang et al.; licensee Beilstein-Institut. \\ License and terms: see end of document.
}

\begin{abstract}
In addition to its well-known abortifacient effect, mifepristone (MIF) has been used as an anticancer drug for various cancers in many studies with an in-depth understanding of the mechanism of action. However, application of MIF is limited by its poor water solubility and low oral bioavailability. In this work, we developed a drug delivery system based on chitosan nanoparticles (CNs) to improve its bioavailability and anticancer activity. The MIF-loaded chitosan nanoparticles (MCNs) were prepared by convenient ionic gelation techniques between chitosan (Cs) and tripolyphosphate (TPP). The preparation conditions, including Cs concentration, TPP concentration, Cs/MIF mass ratio, and $\mathrm{pH}$ value of the TPP solution, were optimized to gain better encapsulation efficiency (EE) and drug loading capacity (DL). MCNs prepared with the optimum conditions resulted in spherical particles with an average size of $200 \mathrm{~nm}$. FTIR and XRD spectra verified that MIF was successfully encapsulated in CNs. The EE and DL of MCNs determined by HPLC were $86.6 \%$ and $43.3 \%$, respectively. The in vitro release kinetics demonstrated that MIF was released from $\mathrm{CNs}$ in a sustained-release manner. Compared with free MIF, MCNs demonstrated increased anticancer activity in several cancer cell lines. Pharmacokinetic studies in male rats that were orally administered MCNs showed a 3.2-fold increase in the area under the curve from 0 to $24 \mathrm{~h}$ compared with free MIF. These results demonstrated that MCNs could be developed as a potential delivery system for MIF to improve its anticancer activity and bioavailability.
\end{abstract}




\section{Introduction}

Mifepristone (RU486, MIF) acts as a progesterone receptor (PR) modulator and has been widely used for emergency contraception and to provoke early-stage abortion [1,2]. Recently, it has been approved by the Federal Drug Administration (FDA) to treat hyperglycemia associated with Cushing's syndrome [3]. Besides its antiglucocorticoid and antiprogestogen activity, MIF has been shown to promote anticancer activity in cancer cell lines and in clinical trials $[4,5]$. However, some side effects of MIF including nausea, vomiting, and bleeding are still observed in the clinic trails [6]. Because the side effects of MIF are closely related with its dosage, some studies have been implemented to find the optimum dosage of MIF [7] or to develop new formulations for MIF to improve its bioavailability [8].

Chitosan (Cs) is a basic polysaccharide found in nature with good biocompatibility and biodegradability [9]. It possesses various bioactivities such as anti-inflammatory, antibacterial, antifungal, muco-adhesive, and antitumor effects [10,11]. Therefore, chitosan has been widely used as a biomaterial or adjuvant in disease therapy [12], tissue engineering, and drug delivery [13]. Owning to the reactive amino side groups, chitosan could be made available via chemical modifications or ionic interactions [14]. Chitosan-bearing protonated amino groups could interact with a wide variety of natural or synthetic anionic species, such as negatively charged proteins, DNA [1519], and some synthetic basic polymers such as sodium tripolyphosphate (TPP) [20,21] to form ionic complexes. This ionic gelation method to prepare Cs/TPP nanoparticles $(\mathrm{CNs})$ with the advantages of simple operation, low equipment requirements, low cost, good repeatability, environmentally friendly, and easy large-scale preparation, has been extensively studied for obtaining nanocarrier systems with a good capacity of drug encapsulation and an adjustable drug release rate $[22,23]$.
The aim of this work was to prepare MIF-encapsulated CNs (MCNs) to regulate the drug release rate of MIF for bioavailability improvement, and meanwhile, enhance the antitumor effect of MIF by the auxiliary anticancer functionality of Cs. The ionic gelation technique was used to prepare MCNs. The preparation conditions for MCNs were optimized and the physiochemical properties of MCNs were characterized. Then, the in vitro drug release behavior of MCNs was determined. Finally, the anticancer activity of MCNs was studied in several cancer cell lines and the pharmacokinetic studies of MCNs were performed in male rats.

\section{Results and Discussion Preparation and optimization of MCNs}

In this study, MIF-loaded CNs were prepared by a convenient ionic gelation technique (Figure 1). This technique has been widely used to prepare CNs as drug delivery systems for a variety of drugs, including either hydrophobic drugs or hydrophilic protein drugs $[22,24]$. Because of the strong hydrophobicity of MIF, it is hard to load MIF into blank CNs after the ionic gelation process. Therefore, Cs was firstly mixed with MIF to afford a homogeneous solution. This solution was then interacted with a TPP solution to allow MIF to be encapsulated during the ionic gelation process. Because Cs was insoluble in water, $2 \%$ aqueous acetic acid solution was used as a solvent for Cs [25]. Ethanol and Tween-80 (1:1 v/v) were used as the organic solvent and detergent, respectively, for MIF because of their low toxic potential and emulsifying activity for dissolution of the hydrophobic components. In the preparation process, it was reported that several factors such as Cs concentration, TPP concentration, Cs/TPP mass ratio could have an influence on particle size, encapsulation efficiency (EE), and drug loading capacity (DL) of the nanoparticles [23,26]. In order to select optimum conditions for preparation of MCNs, the effects of Cs concentration, TPP concentration, and Cs/MIF mass ratio on the

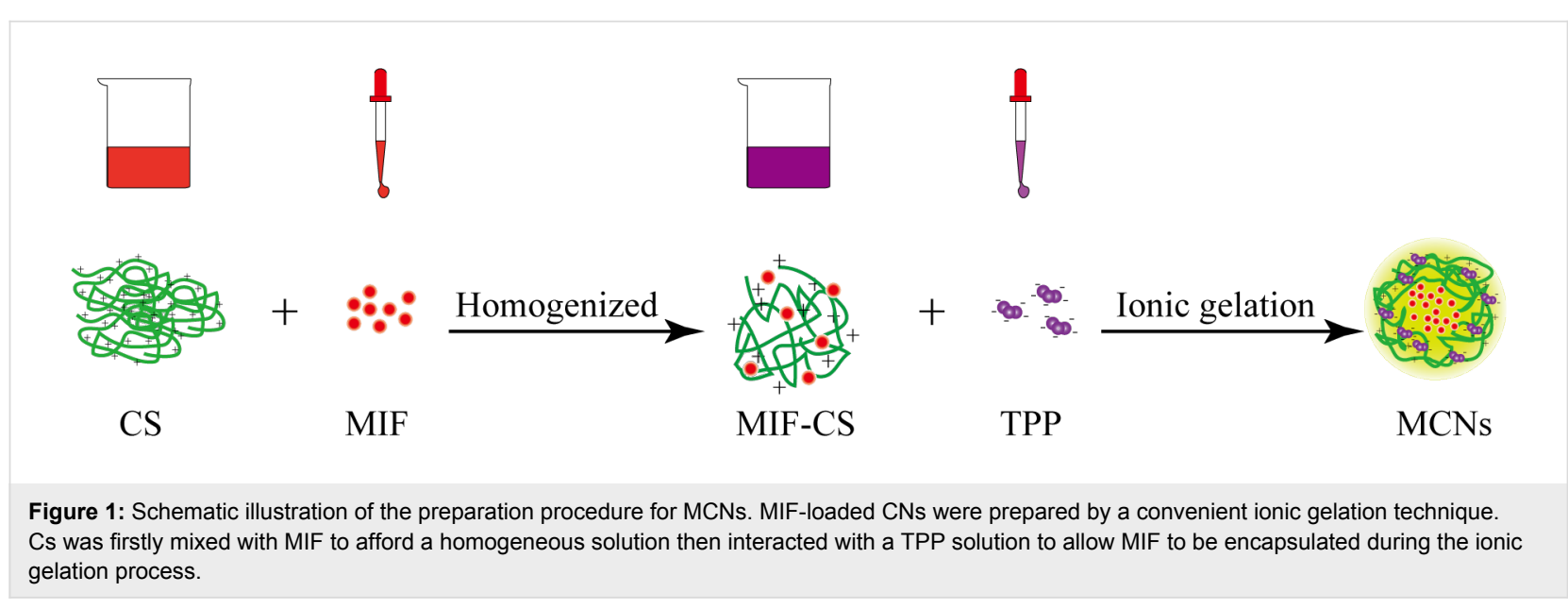


EE and DL of nanoparticles were investigated [25]. Because the $\mathrm{pH}$ values have great influence on the properties of MIF [27], the $\mathrm{pH}$ value of the TPP solution was also optimized.

As shown in Figure 2, the EEs or DLs of different nanoparticle formulations were varied with the change of Cs concentration, TPP concentration, and Cs/MIF mass ratio, and the $\mathrm{pH}$ value of TPP solution. Firstly, we kept the Cs/MIF ratio as 1:1, TPP concentration as $15 \mathrm{mg} / \mathrm{mL}$, and the $\mathrm{pH}$ value of the TPP solution as 7, and investigated the effects of Cs concentration on EE and DL of MCNs. The results showed that a Cs concentration of $12 \mathrm{mg} / \mathrm{mL}$ resulted in higher EE and DL. Cs with low concentration could not entirely entrap MIF, therefore the EE and DL were lower. However, the viscosity of the Cs solution increases with increasing concentration, and the EE will decrease as the degree of dispersion of Cs decreases. In the same way, we found the TPP concentration also has a great effect on EE and DL of MCNs. With the increase of the TPP concentration, the EE and DL of MCNs dropped significantly. This could be due to the increased reaction degree between Cs and TPP, leading to cross-linking of nanoparticles with abnormal morphology, which resulted in decreased EE and DL. The $\mathrm{pH}$ value of the TPP solution is the most important factor affecting EE and DL of MCNs. The MCNs presented the best EE and DL with a $\mathrm{pH}$ 7. This implied better stability and solubility of MIF in neutral $\mathrm{pH}$ conditions. The optimum preparation conditions are: Cs concentration of $12 \mathrm{mg} / \mathrm{mL}, \mathrm{Cs} / \mathrm{MIF}$ ratio of $1: 1$, TPP con- centration of $15 \mathrm{mg} / \mathrm{mL}$, and $\mathrm{pH}$ value of TPP solution of 7 . MCNs prepared with the optimum conditions were then subjected to the following studies.

\section{Characterization}

\section{Fourier transform infrared (FTIR) analysis}

Figure 3 shows the FTIR spectra of the MIF, blank CNs, and the MCNs. There were five characteristic peaks of MIF including a band at $3481 \mathrm{~cm}^{-1}$ due to $-\mathrm{OH}$ stretching vibrations of the

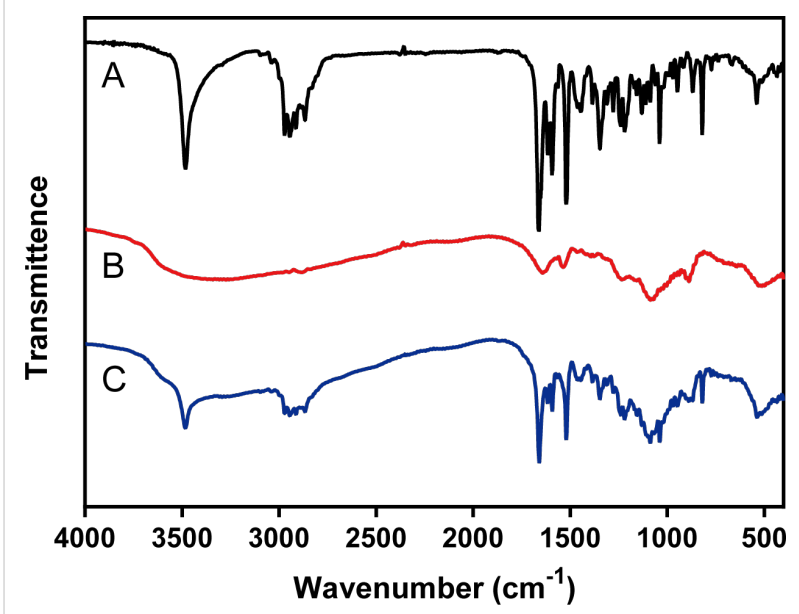

Figure 3: FITR spectra of (A) MIF, (B) blank CNs, and (C) MCNs. The $\mathrm{MCNs}$ formulation showed the characteristic absorption peaks of both MIF and CNs.
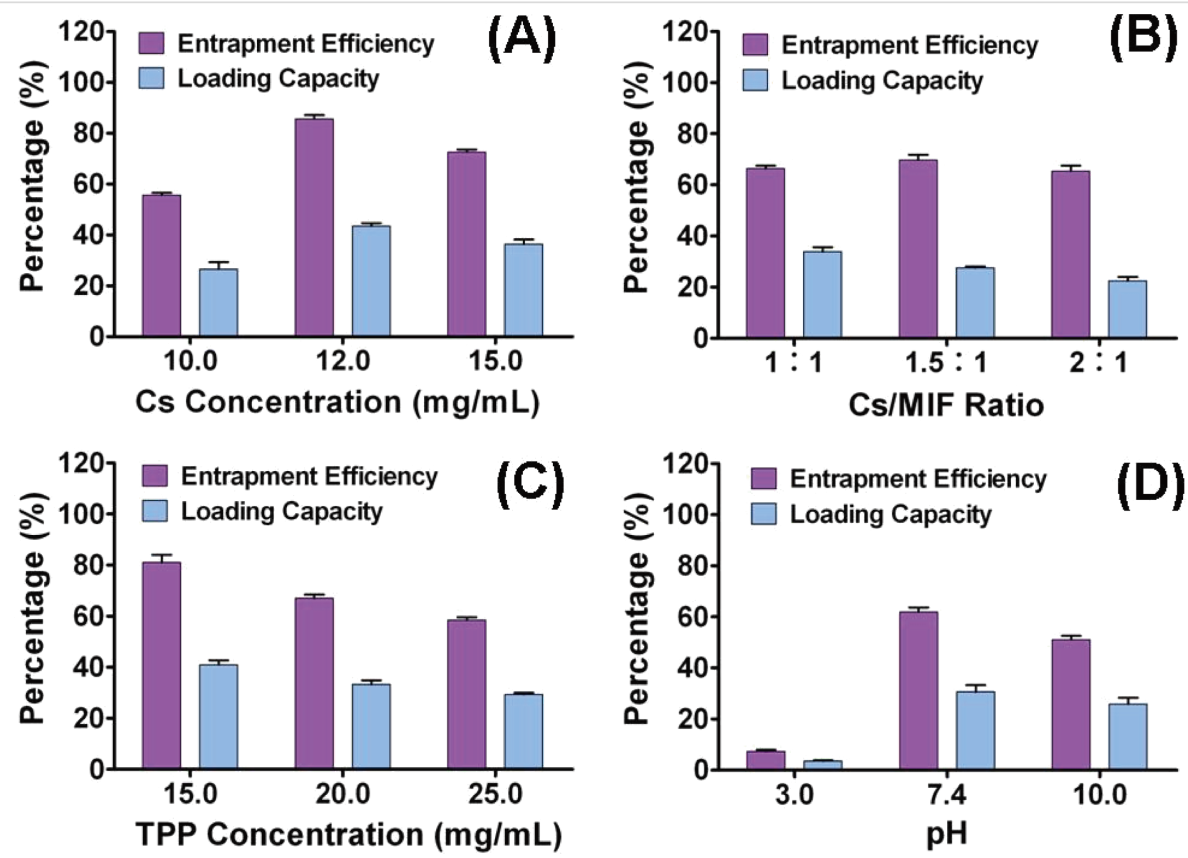

Figure 2: The influence of the preparation conditions including Cs concentration (A), Cs/MIF mass ratio (B), TPP concentration (C), and pH of TPP solution (D) on the encapsulation efficiency (EE) and drug loading capacity (DL) of MCNs. The optimum preparation conditions are: Cs concentration of $12 \mathrm{mg} / \mathrm{mL}$, Cs/MIF mass ratio of $1: 1$, TPP concentration of $15 \mathrm{mg} / \mathrm{mL}$, and TPP solution $\mathrm{pH} 7$. When a parameter is changed, the other variables are selected with the optimum conditions. 
hydroxyl group, a broad band between 2941 and $2866 \mathrm{~cm}^{-1}$ corresponding to the saturated $\mathrm{C}-\mathrm{H}$ stretching vibrations of the various methyl and methylene groups, and two other sharp absorption bands at $1657 \mathrm{~cm}^{-1}$ and $1517 \mathrm{~cm}^{-1}$ corresponding to the $\mathrm{C}-\mathrm{H}$ stretching vibrations of aromatic nucleus. In the FTIR spectrum of blank CNs, characteristic peaks were displayed at $1514 \mathrm{~cm}^{-1}$ due to $+\mathrm{NH}_{3}$ groups [28], and other peaks located at $1086 \mathrm{~cm}^{-1}$ due to $\mathrm{P}-\mathrm{O}$ stretching vibration. The MCNs formulation showed the characteristic absorption peaks of both MIF and CNs, which proved that MIF was successfully wrapped in Cs nanoparticles.

\section{X-ray diffraction (XRD) analysis}

The XRD patterns were studied to observe the change in crystallinity of the MIF in the carrier system of CNs. Figure 4 shows the XRD pattern of the Cs, blank CNs, MIF, and MCNs. In the XRD patterns of Cs (Figure 4A), two intense diffraction peaks of crystallinity were observed. A broadening peak with lower intensity at about $2 \theta$ between $10^{\circ}$ and $30^{\circ}$ was observed in Figure $4 \mathrm{~B}$ as compared with Figure 4A, indicating the decrease of the crystallinity of Cs structure and the presence of $\mathrm{Cs}$ in amorphous forms in the nanoparticles [6]. Figure 4C shows the XRD pattern of MIF, which displayed a distinct spectrum. The XRD analysis of MCNs showed the detailed peaks of MIF superimposed over the broad, amorphous structure of CNs (Figure 4D), indicating that the MIF was embedded in the CNs.

\section{Size and surface morphology of MCNs}

The diameters of CNs and MCNs measured by a Malvern particle size analyzer were found to be from 180 to $200 \mathrm{~nm}$ (Figure 5A). The morphologies of CNs and MCNs were observed using AFM (Figure 5B). It could be seen that the nanoparticles were spherical in shape with a relatively smooth surface. The tiny dots in the AFM images might be due to the free
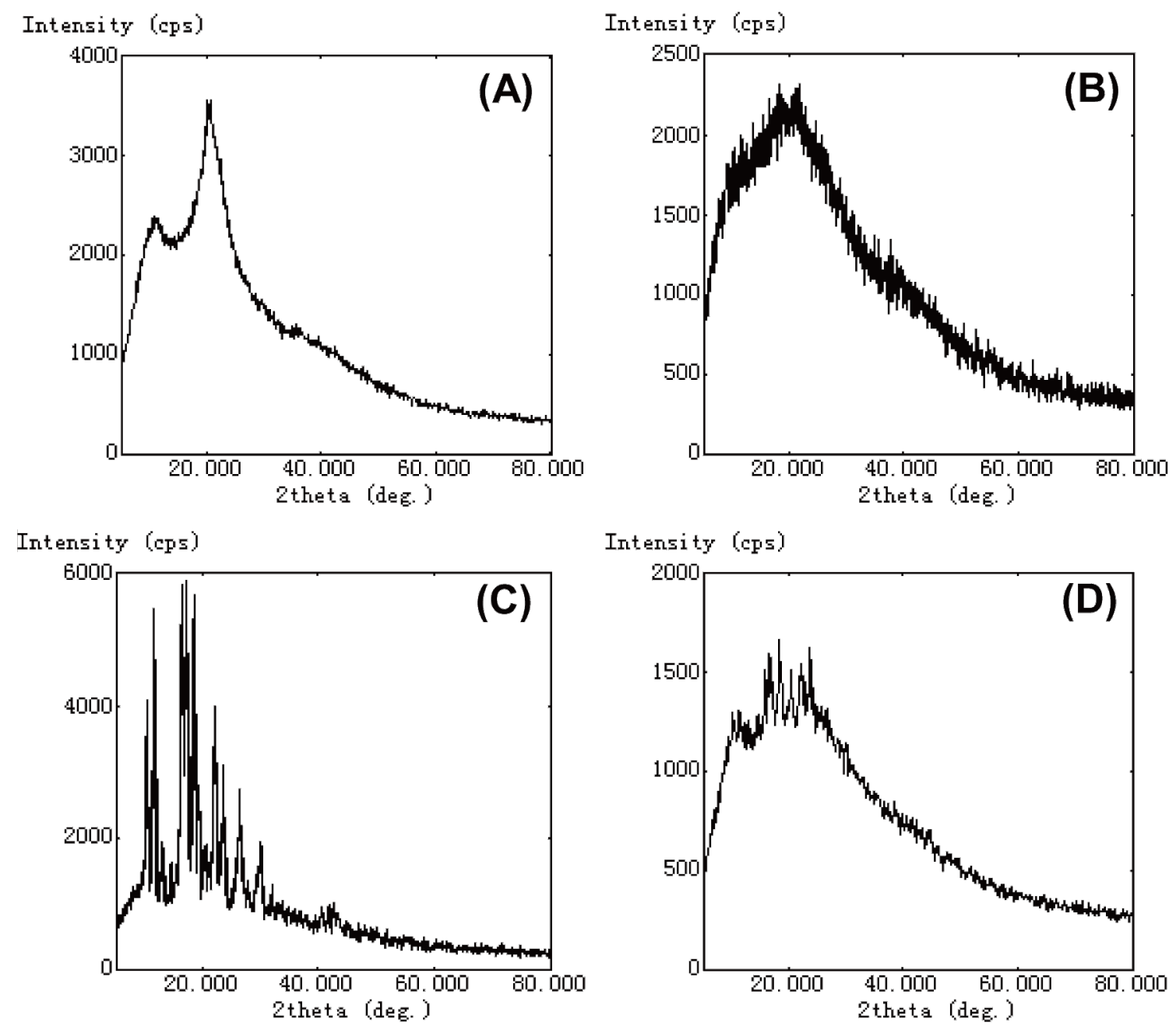

Figure 4: XRD spectra of (A) Cs, (B) blank CNs, (C) MIF, and (D) MCNs. The XRD analysis of MCNs showed the detailed peaks of MIF superimposed over the amorphous features of $\mathrm{CNs}$. 
(A)

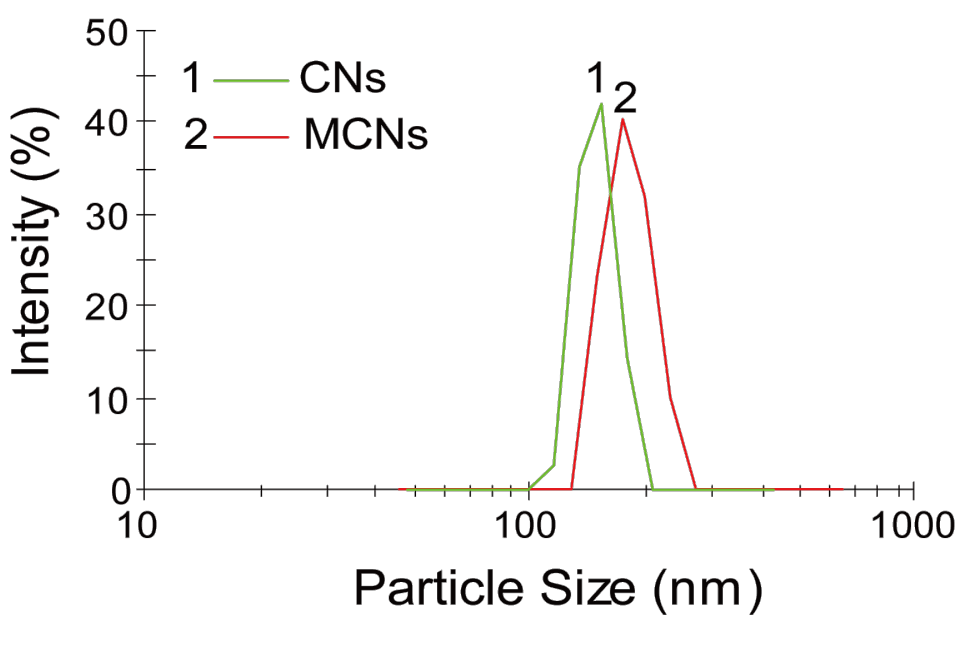

(B)
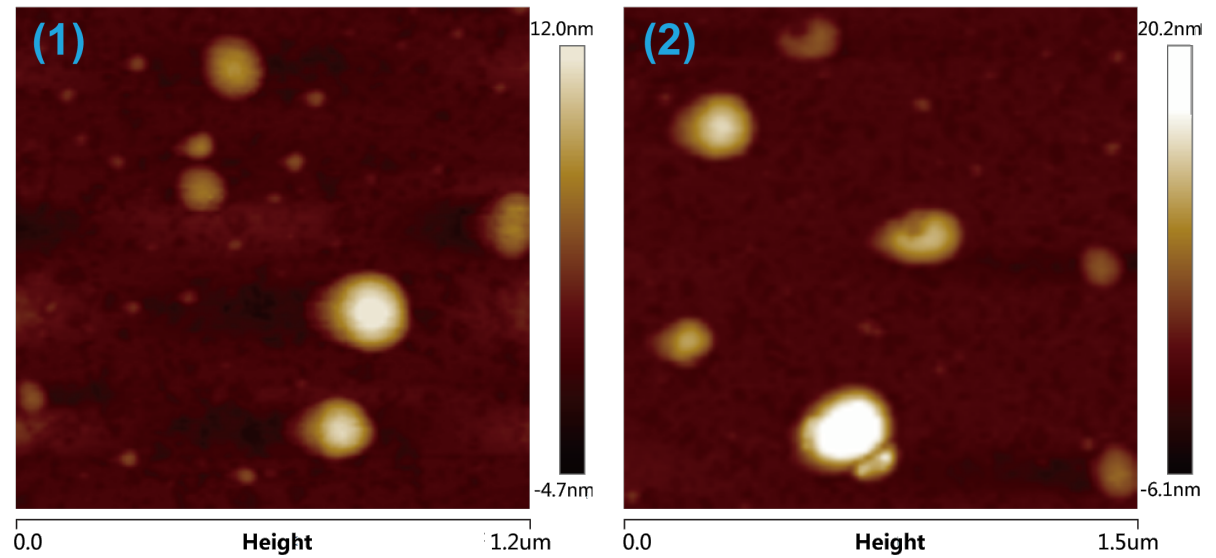

Figure 5: (A) DLS measurements of (1) CNs and (2) MCNs. (B) AFM images of (1) CNs and (2) MCNs.

chitosan. No obvious morphological changes between CNs and MCNs could be found. The results showed that the horizontal distance of MCNs increased from about $150 \mathrm{~nm}$ to $200 \mathrm{~nm}$ and the vertical distance of MCNs increased from about $20 \mathrm{~nm}$ to $30 \mathrm{~nm}$ after MIF entrapment. It was reported that the concentration of TPP and the concentration of Cs have effects on the size of chitosan nanoparticles [26,29]. However, considering that the gastrointestinal absorption and bioavailability of nanoparticles designed for oral administration was closely related to the properties of the composition and the ingredient for specific targeting [30], the influencing factors of the size of MCNs were not studied in this work.

\section{In vitro release study}

The in vitro release study of MIF from MCNs was performed in PBS buffer solutions at $\mathrm{pH} 7.4$ or $\mathrm{pH} 2.5$ to simulate the different $\mathrm{pH}$ conditions of the gastrointestinal system $[31,32]$. To en- hance the solubility of MIF, $1 \%$ of ethanol was added to the incubation medium. The samples were taken out of the medium at designed time points and the released MIF was quantified by HPLC. The release rate of MIF from MCNs showed a sustained release profile in both buffers, and the release rate of MIF from MCNs was very fast at $\mathrm{pH} 2.5$ (Figure 6). This is because MIF, with weakly basic nitrogen, is more likely to dissolve in acidic solution [33]. The sustained-release manner of MCNs could prolong the time of drug absorption in the gastrointestinal tract, which might be beneficial to enhanced bioavailability of MIF $[31,34]$. The sustained-release phenomenon also proved that TPP is an appropriate crosslinking agent for controlled drug release of CNs.

\section{In vitro anticancer effects}

The cytotoxicity of the MCNs was tested in four different cancer cell lines A549 (human lung adenocarcinoma), Hela 


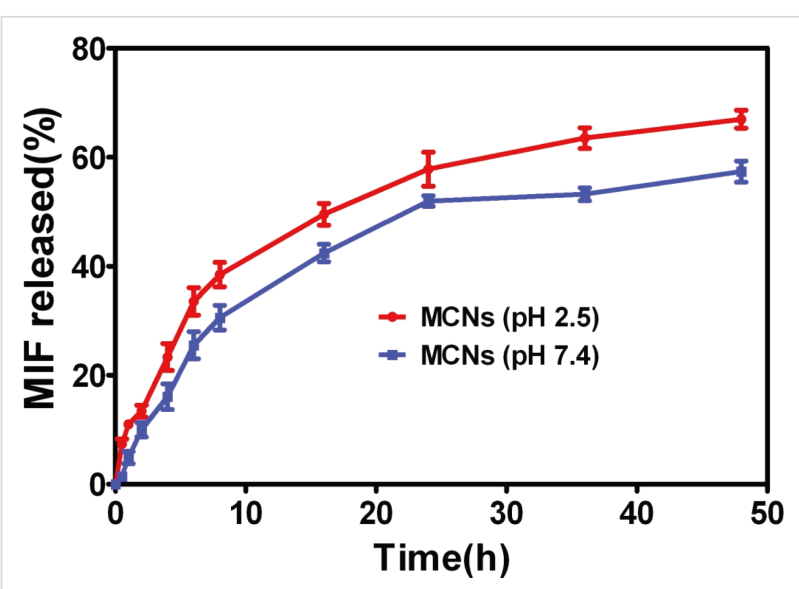

Figure 6: In vitro release profiles of MIF from MCNs at $\mathrm{pH} 2.5$ and 7.4. The release of MIF from the dialysis tube containing MCNs immersed in $0.1 \mathrm{M}$ PBS $(\mathrm{pH} 7.4)$ or $0.1 \mathrm{M}$ PBS $(\mathrm{pH} 2.5)$ containing $1 \%$ of ethanol.

(human cervical epithelioid carcinoma), RL95-2 (human endometrial carcinoma), and HepG2 (human liver hepatocellular carcinoma) cells (Figure 7). CNs demonstrated no obvious toxicity to the four cancer cells under all the tested concentra- tions, indicating that $\mathrm{Cs}$ could be used as a safe drug carrier. However, MCNs could inhibit cell growth in a dose-dependant manner. In this experiment, we found that MIF had no effect at low concentration, and was apt to precipitate at high concentration because of its strong hydrophobicity leading to decreased activity, which was consistent with our previous report [33]. Compared with free MIF, MCNs showed enhanced anti-proliferative activity, indicating that the anticancer effects of MCNs could be due to the synergistic effects of Cs and MIF. Previous studies using other cancer cell lines and other anticancer drugs also found the anticancer effects of Cs and the synergistic interplay of CNs and anticancer drugs [35,36]. Besides, the sustained release of MIF from the CNs could be another reason for the enhanced anti-proliferative efficiency of MCN. In our previous report, we loaded MIF into mesoporous silica nanoparticles (MSNs) coated with aEpCAM (aE-MSN-M) to target circulating tumor cells for cancer metastasis prevention, and also found that MIF entrapped in aE-MSN-M increased its efficacy by sustained release to reduce drug crystallization [33]. These results suggested that MCNs might be a good drug delivery system for delivery of MIF for cancer therapy.
(A)

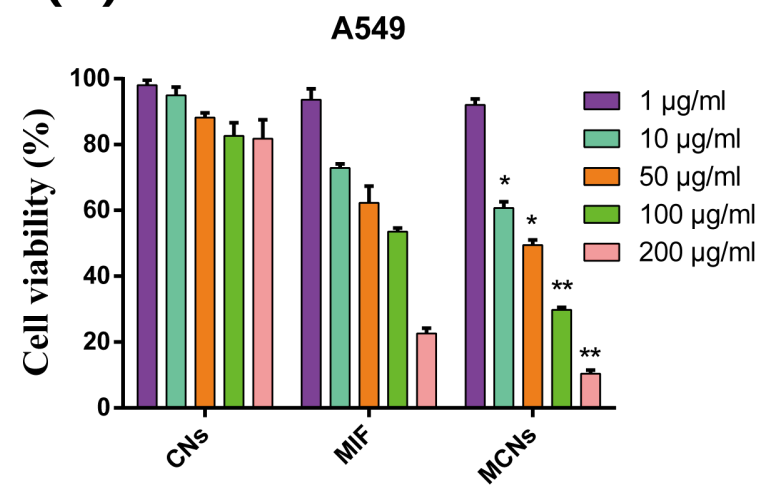

(C)

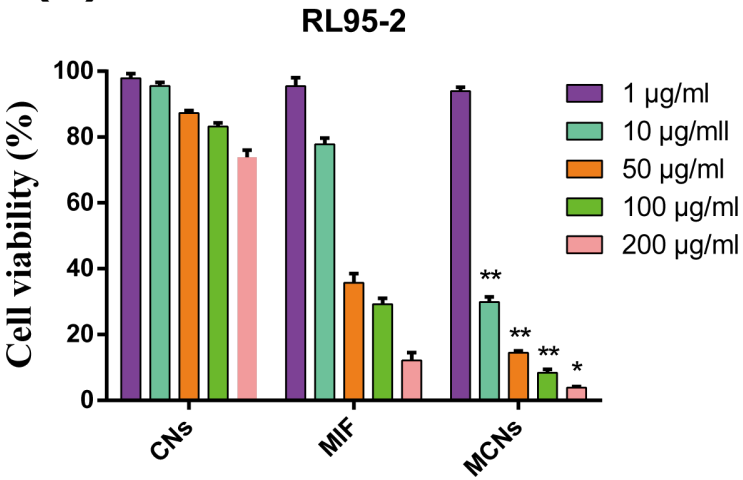

(B)

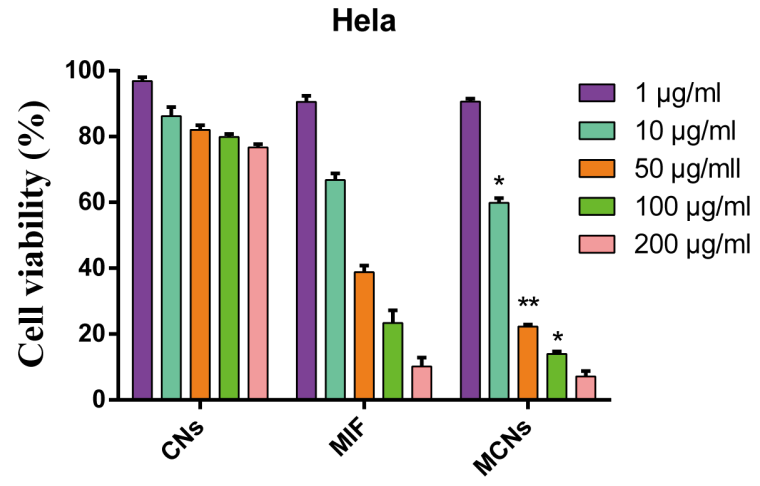

(D)

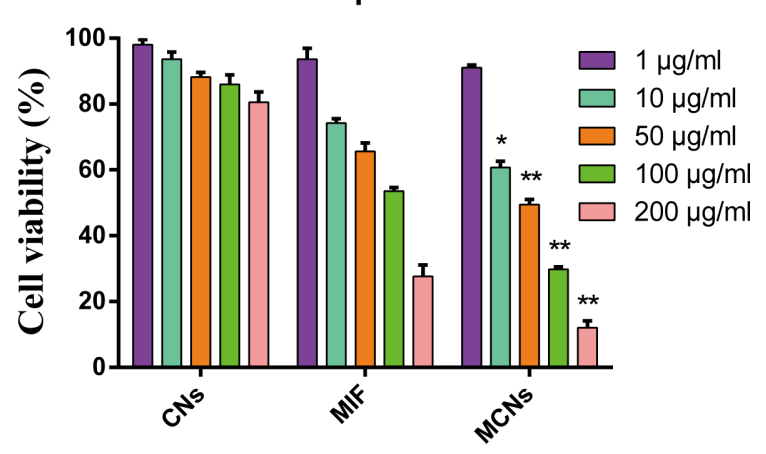

Figure 7: In vitro cytotoxicity of CNs, MIF and MCNs against A549 (A), Hela (B), RL95-2 (C), and HepG2 cells (D). Cells were incubated with different concentrations $(1,10,50,100,200 \mu \mathrm{g} / \mathrm{mL})$ of blank CNs, free MIF, or MCNs for $48 \mathrm{~h}$ at $37^{\circ} \mathrm{C}$ before subjecting them to MTT assay. ${ }^{*} p<0.05$ and ${ }^{* *} p<0.01$ compared with the MIF group by the Student's $t$-test. 


\section{Pharmacokinetic study}

In our previous study, we found MIF showed distinct pharmacokinetic differences between genders. The bioavailability of MIF in male rats was significantly lower than in female rats [37]. Therefore, we chose male rats to perform the pharmacokinetic study to investigate whether MCNs could improve bioavailability of MIF. Following its oral administration, the plasma concentrations of MCNs were compared with pure MIF, and the mean plasma drug concentration-time curves were plotted (Figure 8 ). The corresponding pharmacokinetic parameters including elimination half-life $\left(t_{1 / 2}\right)$, area under the plasma concentration-time curve (AUC), the maximum plasma concentration $\left(C_{\max }\right)$, and the time to maximum plasma concentration $\left(T_{\max }\right)$ are presented in Table 1 . The large error bars in the pharmacokinetics curve MCNs indicated that there are great individual differences in the disposition of MCNs. The statistical analysis indicated that significant differences in $\mathrm{AUC}_{0-t}$ between MCNs and the MIF suspension. The $\mathrm{AUC}_{0-\infty}$ value of the free MIF suspension and the MCNs were $2.4 \pm 0.9 \mathrm{mg} \cdot \mathrm{h} / \mathrm{mL}$

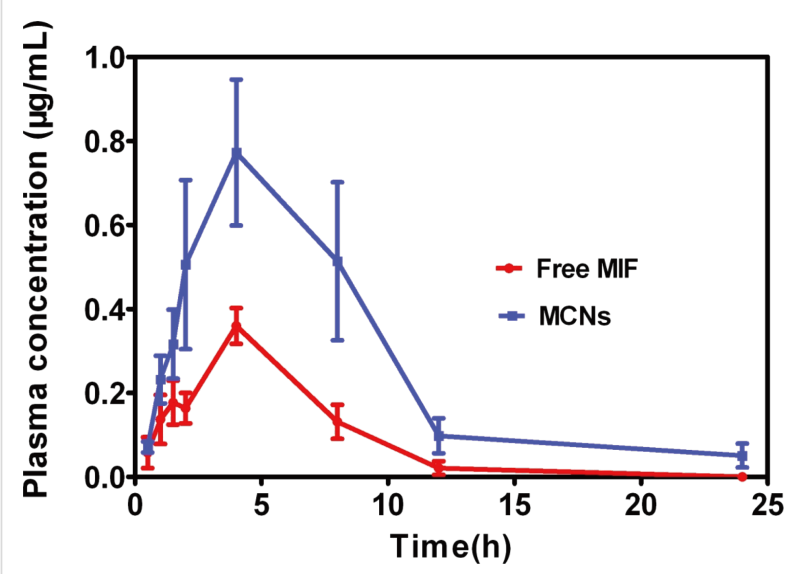

Figure 8: In vivo plasma concentration vs time of different MIF formulations. Male SD rats were given a single $30 \mathrm{mg} / \mathrm{kg}$ dose of MIF (in soybean oil solution) or a single dose of $\mathrm{MCNs}$ equivalent to the same dosage of MIF. Blood samples (each $0.5 \mathrm{~mL}$ ) were collected into heparinized tubes from the orbital venous plexus at different time points after oral administration.

Table 1: Pharmacokinetic parameters of different MIF formulations.

\begin{tabular}{lll} 
Parameters & MIF suspension & MCNs \\
\hline $\mathrm{AUC}_{0-t}(\mathrm{mg} / \mathrm{L} \cdot \mathrm{h})$ & $2.0 \pm 0.5$ & $6.3 \pm 3.8^{\mathrm{a}}$ \\
$\mathrm{AUC}_{0-\infty}(\mathrm{mg} / \mathrm{L} \cdot \mathrm{h})$ & $2.4 \pm 0.9$ & $6.8 \pm 4.3^{\mathrm{b}}$ \\
$t_{1 / 2}(\mathrm{~h})$ & $3.0 \pm 2.0$ & $4.0 \pm 2.8$ \\
$T_{\max }(\mathrm{h})$ & $3.4 \pm 1.2$ & $5.0 \pm 2.0$ \\
$C_{\max }(\mathrm{mg} / \mathrm{L})$ & $0.36 \pm 0.09$ & $0.79 \pm 0.33^{* *}$ \\
\hline
\end{tabular}

${ }^{a} p<0.05 ;{ }^{b} p<0.01$ as compared with the MIF group by Student's $t$-test. and $6.8 \pm 4.3 \mathrm{mg} \cdot \mathrm{h} / \mathrm{mL}$, respectively. In addition, the $t_{1 / 2}$ of the MCN group was longer than the MIF suspension group. These results demonstrated a relatively high and effective absorption of MCNs in vivo. The large surface area produced by the nanoparticles and the bioadhesive properties of Cs allow more of the drug to interact with the gastrointestinal tract $[38,39]$. These pharmacokinetic data clearly evidence the ability of CNs to enhance the absorption of MIF, which suggests that CNs could be a good formulation for MIF to increase its relative bioavailability.

\section{Conclusion}

In conclusion, CNs were employed as a drug delivery system for MIF delivery to improve the bioavailability of MIF, and consequently, to enhance the antitumor effect of MIF by the auxiliary anticancer functionality of Cs. MCNs prepared by an ionic gelation method under optimum preparation conditions were spherical in shape with an average diameter of $200 \mathrm{~nm}$ and satisfied EE and DL. FTIR and XRD spectra verified that MIF was successfully encapsulated in CNs. MIF could be released from the $\mathrm{CNs}$ in a sustained-release manner. MCNs were shown to increase the anticancer activity of MIF in several cancer cell lines and improved the oral absorption of MIF in male rats. All these results suggest that the MCNs may be further developed as a potential delivery system for MIF for cancer therapy.

\section{Experimental Materials}

Chitosan (deacetylation degree of $90 \%$ and $\mathrm{MW}=60 \mathrm{kDa}, \mathrm{Cs}$ ) was purchased from Boao Biotechnology Co., Ltd. (Shanghai, China). Sodium tripolyphosphate (TPP) was purchased from Aladdin Industrial Co., Ltd. (Shanghai, China). Tween-80 and ethanol were purchased from Sinopharm Chemical Reagent Co., Ltd. (Shanghai, China). The RPMI 1640 medium, Dulbeccos Modified Eagle Medium (DMEM), McCoy's 5A medium, antibiotics, and fetal bovine serum (FBS) were purchased from Life Technologies GmbH (Darmstadt, Germany). 3-(4,5-Dimethylthiazol-2-yl)-2,5-diphenyltetrazolium bromide (MTT) was obtained from Sigma-Aldrich. Mifepriston (MIF) with the purity $>98 \%$ was provided by Shanghai New Hualian Pharmaceutical Co., Ltd. (Shanghai, China). All other solvents and chemicals used were of analytical grade.

\section{Preparation of MCNs}

Briefly, Cs was dissolved in 2\% (w/v) acetic acid solution and the solution was left standing for $1 \mathrm{~h}$ before use. MIF was dissolved in mixed solution of anhydrous ethanol and Tween-80 $(1: 1 \mathrm{v} / \mathrm{v})$ and added to the Cs solution at different Cs/MIF mass ratios. After fully degassing, the resulting mixture was added dropwise into agitated TPP solution $(0.3 \% \mathrm{w} / \mathrm{v})$ at different $\mathrm{pH}$ values and the suspension was stirred at room temperature for 
$30 \mathrm{~min}$. After standing for $2 \mathrm{~h}$, the resulting complex was filtered out, washed at least three times with deionized water to remove the residual TPP, and dried in a desiccator to keep the weight constant. The collected MCNs were ground and stored at $4{ }^{\circ} \mathrm{C}$ for further research. The blank CNs were prepared similarly without adding MIF.

\section{Characterization}

Infrared spectra were analyzed using a FTIR spectrometer (Intelligent, Nicolet 360, USA) with KBr pellet. The samples were scanned from $500-4000 \mathrm{~cm}^{-1}$ (scan step of $4 \mathrm{~cm}^{-1}$ ).

X-ray powder diffraction patterns of MCNs were obtained by an XRD diffractometer (Miniflex II, Rigaku, Japan). The X-ray source was $\mathrm{Cu} \mathrm{K} \alpha$ radiation $(30 \mathrm{kV}, 15 \mathrm{~mA})$.

AFM images were obtained on a Multimode 8 AFM series (Bruker, USA) in tapping/AC mode. The mean particle size and zeta potential of the MCNs were determined by dynamic light scattering using a Nanotrac ${ }^{\circledR}$ Wave Particle Size and Zeta Potential Analyzer (Microtrac Inc, Montgomeryville, PA).

\section{Encapsulation efficiency (EE) and drug loading (DL) capacity}

The EE and the DL of MIF in the MCNs was analyzed by high performance liquid chromatography (HPLC). Briefly, an accurately weighed quantity of the MCN powder was suspended in a specified volume of anhydrous alcohol with vigorous stirring for a period of time. The supernatant was collected after centrifugation and the concentration of MIF was analyzed with a Waters-2695 HPLC system equipped with a 2487 UV detector. MIF was separated on an Inertstil ODS2 C18 $(150 \times 4.6 \mathrm{~mm}, 5 \mu \mathrm{m})$ column with the mobile phase of acetonitrile-water $(80: 20 \mathrm{v} / \mathrm{v})$, injection volume of $20 \mu \mathrm{L}$, flow rate of $1.0 \mathrm{~mL} / \mathrm{min}$, ambient column temperature and detection wavelength of $302 \mathrm{~nm}$ [33].

The EE and DL of MCNs were analyzed using the following formulas:

$$
\begin{aligned}
& \mathrm{EE}(\%)=\frac{\text { Amount of MIF in nanoparticles }}{\text { Total amount of MIF added }} \times 100 \\
& \mathrm{DL}(\%)=\frac{\text { Amount of MIF in nanoparticles }}{\text { Amount of nanoparticles }} \times 100
\end{aligned}
$$

\section{In vitro drug release study}

The in vitro release of MIF from the MCNs was performed using the dialysis bag diffusion technique [33]. The dialysis bag was cut into small pieces of appropriate length and boiled for $10 \mathrm{~min}$ in a volume $(500 \mathrm{~mL})$ of $2 \%(\mathrm{w} / \mathrm{v})$ sodium bicarbonate and $1 \mathrm{mmol} / \mathrm{L}$ EDTA-2Na (pH 8.0) before use. Briefly, $10 \mathrm{mg}$ of dried MCNs were resuspended in $10 \mathrm{~mL}$ of dissolution media; the suspension was then put into a pretreated dialysis bag which was sealed with a dialysis bag holder. The sealed dialysis bag was put into a large beaker containing $500 \mathrm{~mL} 1 \%$ ethanol/PBS solution of $\mathrm{pH} 7.4$ or $\mathrm{pH} 2.5$. The solution was stirred with a magnetic stirrer at $37{ }^{\circ} \mathrm{C}$ under a light-sealed condition. At certain time intervals, $1 \mathrm{~mL}$ of the release medium was taken out and the concentration of the released MIF was determined based on a free MIF calibration curve using the HPLC method as described above.

\section{In vitro anti-proliferative activity \\ Cell lines and cell culture}

A549 human lung cancer cells, human epithelial carcinoma Hela cells, human endometrial carcinoma RL95-2 cells, and human hepatocellular liver carcinoma HepG2 cells were purchased from Type Culture Collection of the Chinese Academy of Sciences (Shanghai, China). A549 and Hela was grown in DMEM containing $10 \%$ FBS, $100 \mathrm{U} / \mathrm{mL}$ penicillin $\mathrm{G}$ sodium and $100 \mu \mathrm{g} / \mathrm{mL}$ streptomycin sulfate. RL95-2 and HepG2 cells were cultured in RMPI1640 mixed with 10\% FBS (v/v), $100 \mathrm{U} / \mathrm{mL}$ penicillin G sodium, and $100 \mu \mathrm{g} / \mathrm{mL}$ streptomycin sulfate. The cells were incubated at $37{ }^{\circ} \mathrm{C}$ with $5 \% \mathrm{CO}_{2}$ in a humid cell incubator.

\section{MTT assay}

A549, Hela, RL95-2 and HepG2 cells were seeded in a 96-well plate (8000 per well) and incubated for $24 \mathrm{~h}$ at $37{ }^{\circ} \mathrm{C}$ with $5 \%$ $\mathrm{CO}_{2}$. Blank CNs, free MIF, or MCNs were added to the well at predetermined drug concentrations $(1,10,50,100,200 \mu \mathrm{g} / \mathrm{mL})$, and incubated for $48 \mathrm{~h}$ at $37{ }^{\circ} \mathrm{C}$ with $5 \% \mathrm{CO}_{2}$. The medium was removed and the cells were washed three times with PBS before incubation with MTT for $4 \mathrm{~h}$ at $37^{\circ} \mathrm{C}$. After the medium was removed, $100 \mu \mathrm{L}$ of DMSO was added to the well for $20 \mathrm{~min}$. The amount of MTT formazan product was analyzed spectrophotometrically at $570 \mathrm{~nm}$ by a TECAN Infinite F200 microplate reader.

\section{Pharmacokinetic study \\ Animals}

Male Sprague-Dawley (SD) rats (180-220 g) were supplied by the Experimental Animal Center of Zhejiang Province and were housed with a $12 \mathrm{~h}$ dark/light cycle for three days before starting the experiment. The rats were fed a standard diet with water to drink ad libitum. Before drug administration, the rats were fasted overnight with free access to water. All studies involving animals were carried out in accordance with the National Nature Science Foundation of China (NSFC) regulation concerning the care and use of experimental animals and approved by our Animal Care and Use Committee to reduce the suffering and use of animals. 


\section{Oral administration}

Eight male SD rats were randomly divided into two groups $(n=4)$. Group 1 was given a single $30 \mathrm{mg} / \mathrm{kg}$ dose of MIF (in soybean oil solution) and Group 2 was given a single dose of MCNs equivalent to the same dosage of MIF. Blood samples (each $0.5 \mathrm{~mL}$ ) were collected in heparinized tubes from the orbital venous plexus at $0.5,1,1.5,2,4,8,12$, and $24 \mathrm{~h}$ after oral administration. All blood samples were immediately processed by centrifugation at $4000 \mathrm{rpm}$ for $8 \mathrm{~min}$, and the plasma samples were stored at $-20{ }^{\circ} \mathrm{C}$ before analysis.

\section{Blood sample preparation}

After the frozen plasma sample was thawed to room temperature, an aliquot of $200 \mu \mathrm{L}$ of plasma was spiked with $50 \mu \mathrm{L}$ levonorgestrel (internal standard, I.S., $98.0 \%$ purity) solution $(426 \mathrm{ng} / \mathrm{mL})$ in a $1.5 \mathrm{~mL}$ centrifuge tube and homogenized by vortex-mixing for $3 \mathrm{~min}$. The mixed sample was then extracted with $2.0 \mathrm{~mL}$ of ethyl acetate by vortex-mixing for $3 \mathrm{~min}$. After centrifugation at $4000 \mathrm{rpm}$ for $10 \mathrm{~min}$, the upper separated organic layer was carefully collected and evaporated to dryness under a gentle stream of nitrogen gas at $50{ }^{\circ} \mathrm{C}$. The dried residue was reconstituted in $100 \mu \mathrm{L}$ of methanol-water solution $(50: 50 \mathrm{v} / \mathrm{v})$ followed by vortex-mixing for $3 \mathrm{~min}$ and then centrifuged at 15,000 rpm for $10 \mathrm{~min}$. Afterwards, a $4 \mu \mathrm{L}$ aliquot of the supernatant was injected into the chromatographic systems for analysis.

\section{Quantification}

The MIF concentration in plasma was determined using LC-MS/MS analysis according to the method reported earlier by Chen et al. [37] with slight modifications. Liquid chromatography was performed on an ACQUITY UPLC system using a BEH $\mathrm{C}_{18}$ column $(50 \mathrm{~mm} \times 2.1 \mathrm{~mm}, 1.7 \mu \mathrm{m}$, Waters Corporation, USA). The mobile phase solution was composed of methanol (A) and aqueous $0.1 \%(\mathrm{v} / \mathrm{v})$ formic acid (B) with a gradient program as follows: $0-1.0 \mathrm{~min}(40-95 \% \mathrm{~A}), 1.0-2.5 \mathrm{~min}$ (95-95\% A), 2.5-2.8 $\min (95-40 \%$ A), 2.8-4.0 $\min (40-40 \%$ A). The column temperature and flow rate were $35{ }^{\circ} \mathrm{C}$ and $0.3 \mathrm{~mL} / \mathrm{min}$, respectively. The injection volume was $4 \mu \mathrm{L}$.

The mass spectrometer (Waters Corporation., Milford, MA, USA) was operated in positive mode and equipped with an electrospray ionization (ESI) source. The main operating parameters were optimized as follows: desolvation gas (nitrogen) $600 \mathrm{~L} / \mathrm{h}$, cone gas (nitrogen) $50 \mathrm{~L} / \mathrm{h}$, collision gas (argon) about $0.15 \mathrm{MPa}$, cone voltage $30 \mathrm{~V}$, capillary voltage $3.2 \mathrm{kV}$, source temperature $110{ }^{\circ} \mathrm{C}$, and desolvation temperature $350{ }^{\circ} \mathrm{C}$. The detection was operated in the multiple reaction monitoring (MRM) mode, and the MRM transitions were $m / z 430.2 \rightarrow 134.0$ for MIF, and $m / z 313.3 \rightarrow 109.0$ for I.S., respectively.
MIF was found stable in plasma under the stability test conditions. The calibration curve exhibited good linearity in the range of 7.1-2840 ng/mL $\left(R^{2}>0.998\right)$. The calibration curves were fitted with a weighted $\left(1 / \chi^{2}\right)$ least-squares linear regression method. A typical regression equation for the calibration curve was $y=2.66736 \chi+0.110667, R^{2}=0.9980$. The average recovery of MIF from the isolated plasma solution was greater than $80 \%$.

\section{Pharmacokinetic parameters and statistics}

Pharmacokinetic parameters were calculated by using DAS version 3.0 software (BioGuider Co., Shanghai, China) including elimination half-life $\left(t_{1 / 2}\right)$, and area under the plasma concentration-time curve (AUC). The maximum plasma concentration $\left(C_{\max }\right)$ and the time to maximum plasma concentration $\left(T_{\max }\right)$ were directly read from the experimental data. Statistical analyses of pharmacokinetic parameters were performed by ANOVA to analyze differences among groups with $p<0.05$ as the level of significance.

\section{Pharmacokinetic study}

Statistical analysis was performed using the Student's $t$-test. The differences were considered significant for $p<0.05$, and $p<0.01$ was indicative of a very significant difference.

\section{Acknowledgements}

This work was supported by the Ministry of Science and Technology of China (2015CB931804), Natural Science Foundation of China (81571802 and 81402781), Natural Science Foundation of Fujian Province (2016J06020), the Scientific Research Foundation for the Returned Overseas Chinese Scholars, Fujian Development and Reform Commission (2014/ 168), and Zhejiang Provincial Natural Science Foundation (LQ14B020004).

\section{References}

1. Kekkonen, R.; Heikinheimo, O.; Mandelin, E.; Lähteenmäki, P. Contraception 1996, 54, 229-234. doi:10.1016/S0010-7824(96)00193-X

2. Mahajan, D. K.; London, S. N. Fertil. Steril. 1997, 68, 967-976. doi:10.1016/S0015-0282(97)00189-1

3. Carmichael, J. D.; Fleseriu, M. Endocrine 2013, 44, 20-32. doi:10.1007/s12020-012-9846-1

4. Chen, J.; Wang, J.; Shao, J.; Gao, Y.; Xu, J.; Yu, S.; Liu, Z.; Jia, L. Med. Res. Rev. 2014, 34, 979-1000. doi:10.1002/med.21311

5. Fleseriu, M.; Biller, B. M. K.; Findling, J. W.; Molitch, M. E.; Schteingart, D. E.; Gross, C.; SEISMIC Study Investigators. J. Clin. Endocrinol. Metab. 2012, 97, 2039-2049. doi:10.1210/jc.2011-3350

6. Sarkar, N. N. Eur. J. Obstet. Gynecol. Reprod. Biol. 2002, 101, 113-120. doi:10.1016/S0301-2115(01)00522-X

7. Liao, A.; Pang, X.; Li, H.; Xiong, Z.; Wu, X. Contraception 2008, 77, 431-434. doi:10.1016/j.contraception.2008.02.009 
8. He, W.; Horn, S. W.; Hussain, M. D. Int. J. Pharm. 2007, 334, 173-178. doi:10.1016/j.ijpharm.2006.10.025

9. Choi, C.; Nam, J.-P.; Nah, J.-W. J. Ind. Eng. Chem. 2016, 33, 1-10. doi:10.1016/j.jiec.2015.10.028

10. Ong, S.-Y.; Wu, J.; Moochhala, S. M.; Tan, M.-H.; Lu, J. Biomaterials 2008, 29, 4323-4332. doi:10.1016/j.biomaterials.2008.07.034

11. Croisier, F.; Jérôme, C. Eur. Polym. J. 2013, 49, 780-792. doi:10.1016/j.eurpolymj.2012.12.009

12. Przekora, A.; Palka, K.; Ginalska, G. Mater. Sci. Eng., C 2016, 58, 891-899. doi:10.1016/j.msec.2015.09.046

13. Park, G. H.; Kang, M.-S.; Knowles, J. C.; Gong, M.-S. J. Biomater. Appl. 2016, 30, 1350-1361. doi:10.1177/0885328215626892

14. Zargar, V.; Asghari, M.; Dashti, A. ChemBioEng Rev. 2015, 2, 204-226. doi:10.1002/cben.201400025

15. Strand, S. P.; Lelu, S.; Reitan, N. K.; de Lange Davies, C.; Artursson, P.; Vårum, K. M. Biomaterials 2010, 31, 975-987. doi:10.1016/j.biomaterials.2009.09.102

16. Gao, P.; Xia, G.; Bao, Z.; Feng, C.; Cheng, X.; Kong, M.; Liu, Y.; Chen, X. Int. J. Biol. Macromol. 2016, 91, 716-723. doi:10.1016/j.jjbiomac.2016.06.015

17. Gao, Y.; Zhang, Z.; Chen, L.; Gu, W.; Li, Y. Biomacromolecules 2009, 10, 2175-2282. doi:10.1021/bm900341d

18. Gao, Y.; Zhang, Z.; Chen, L.; Gu, W.; Li, Y. Int. J. Pharm. 2009, 371, 156-162. doi:10.1016/j.ijpharm.2008.12.012

19. Gao, Y.; Xu, Z.; Chen, S.; Gu, W.; Chen, L.; Li, Y. Int. J. Pharm. 2008, 359, 241-246. doi:10.1016/j.ijpharm.2008.03.037

20. Cho, Y.; Shi, R.; Borgens, R. B. J. Biol. Eng. 2010, 4, 2. doi:10.1186/1754-1611-4-2

21. Nguyen, T. V.; Nguyen, T. T. H.; Wang, S.-L.; Vo, T. P. K.; Nguyen, A. D. Res. Chem. Intermed. 2016, 1-11. doi:10.1007/s11164-016-2428-8

22. Jain, A.; Thakur, K.; Sharma, G.; Kush, P.; Jain, U. K. Carbohydr. Polym. 2016, 137, 65-74. doi:10.1016/j.carbpol.2015.10.012

23. Hashad, R. A.; Ishak, R. A.; Fahmy, S.; Mansour, S.; Geneidi, A. S. Int. J. Biol. Macromol. 2016, 86, 50-58. doi:10.1016/j.ijbiomac.2016.01.042

24. Azevedo, J. R.; Sizilio, R. H.; Brito, M. B.; Costa, A. M. B.; Serafini, M. R.; Araújo, A. A. S.; Santos, M. R. V.; Lira, A. A. M.; Nunes, R. S. J. Therm. Anal. Calorim. 2011, 106, 685-689. doi:10.1007/s10973-011-1429-5

25. Wang, L.-Y.; Gu, Y.-H.; Su, Z.-G.; Ma, G.-H. Int. J. Pharm. 2006, 311, 187-195. doi:10.1016/j.ijpharm.2005.12.033

26. de Pinho Neves, A. L.; Milioli, C. C.; Müller, L.; Riella, H. G.; Kuhnen, N. C.; Stulzer, H. K. Colloids Surf., A 2014, 445, 34-39. doi:10.1016/j.colsurfa.2013.12.058

27. Guo, Z.; Wei, D.; Yin, G.; Wang, S.; Zhao, S.; Chu, Y.; Zhai, J. J. Chromatogr., B 2007, 856, 312-317. doi:10.1016/j.jchromb.2007.06.016

28. Zhang, C.; Ping, Q.; Ding, Y.; Cheng, Y.; Shen, J. J. Appl. Polym. Sci. 2003, 91, 659-665. doi:10.1002/app.13232

29. Ko, J. A.; Park, H. J.; Hwang, S. J.; Park, J. B.; Lee, J. S. Int. J. Pharm. 2002, 249, 165-174. doi:10.1016/S0378-5173(02)00487-8

30. Li, Z.; Jiang, H.; Xu, C.; Gu, L. Food Hydrocolloids 2015, 43, 153-164. doi:10.1016/j.foodhyd.2014.05.010

31. Lal, S.; Datta, M. Appl. Clay Sci. 2015, 114, 412-421. doi:10.1016/j.clay.2015.06.017
32. Shah, B. R.; Li, Y.; Jin, W.; An, Y.; He, L.; Li, Z.; Xu, W.; Li, B. Food Hydrocolloids 2016, 52, 369-377. doi:10.1016/j.foodhyd.2015.07.015

33. Gao, Y.; Gu, S.; Zhang, Y.; Xie, X.; Yu, T.; Lu, Y.; Zhu, Y.; Chen, W.; Zhang, H.; Dong, H.; Sinko, P. J.; Jia, L. Small 2016, 12, 2595-2608. doi:10.1002/smll.201600550

34. Fang, G.; Tang, B.; Chao, Y.; Zhang, Y.; Xu, H.; Tang, X. RSC Adv. 2015, 5, 96437-96447. doi:10.1039/C5RA14588K

35. Gibot, L.; Chabaud, S.; Bouhout, S.; Bolduc, S.; Auger, F. A.; Moulin, V. J. Int. J. Biol. Macromol. 2015, 72, 370-379. doi:10.1016/j.ijbiomac.2014.08.033

36. Kandra, P.; Kalangi, H. P. J. Appl. Microbiol. Biotechnol. 2015, 99 , 2055-2064. doi:10.1007/s00253-015-6384-9

37. Chen, J.-Z.; Wang, J.-C.; Gao, Y.; Zeng, R.-J.; Jiang, Z.; Zhu, Y.-W.; Shao, J.-W.; Jia, L. J. Pharm. Biomed. Anal. 2014, 95, 158-163. doi:10.1016/j.jpba.2014.02.026

38. Eldridge, J. H.; Hammond, C. J.; Meulbroek, J. A.; Staas, J. K.; Gilley, R. M.; Tice, T. R. J. Controlled Release 1990, 11, 205-214. doi:10.1016/0168-3659(90)90133-E

39. Galindo-Rodriguez, S. A.; Allemann, E.; Fessi, H.; Doelker, E. Crit. Rev. Ther. Drug Carrier Syst. 2005, 22, 419-464. doi:10.1615/CritRevTherDrugCarrierSyst.v22.i5.10

\section{License and Terms}

This is an Open Access article under the terms of the Creative Commons Attribution License (http://creativecommons.org/licenses/by/4.0), which permits unrestricted use, distribution, and reproduction in any medium, provided the original work is properly cited.

The license is subject to the Beilstein Journal of

Nanotechnology terms and conditions:

(http://www.beilstein-journals.org/bjnano)

The definitive version of this article is the electronic one which can be found at: $\underline{\text { doi: } 10.3762 / \text { bjnano. } 7.178}$ 